\title{
An immunohistochemical study of cyclin- dependent kinase 5 (CDK5) expression in non-small cell lung cancer (NSCLC) and small cell lung cancer (SCLC): a possible prognostic biomarker
}

Kanglai Wei ${ }^{1 \dagger}$, Zhihua Ye ${ }^{1 \dagger}$, Zuyun $\mathrm{Li}^{1}$, Yiwu Dang ${ }^{1}$, Xin Chen ${ }^{1}$, Na Huang ${ }^{1}$, Chongxi Bao ${ }^{1}$, Tingqing Gan², Lihua Yang ${ }^{2^{*}}$ and Gang Chen ${ }^{1^{*}}$

\begin{abstract}
Background: Cyclin-dependent kinase 5 (CDK5) is an atypical CDK which plays a vital role in several cancers via regulating migration and motility of cancer cells. However, the clinicopathological impact and function of CDK5 in lung cancer remain poorly understood. The present study was aimed at exploring expression and clinicopathological significance of CDK5 in lung cancer.

Methods: There were 395 samples of lung tissue including 365 lung tumors (339 non-small cell lung cancers and 26 small cell lung cancers) and 30 samples of normal lung. CDK5 expression was detected by immunohistochemistry on lung tissue microarrays.

Results: Over expression was detected in lung cancer compared with normal lung tissues $(P=0.001)$. Furthermore, area under curve (AUC) of receiver operating characteristic (ROC) of CDK5 was 0.685 (95\% Cl 0.564 0.751, $P=0.004$ ). In lung cancer, we also discovered close correlations between CDK5 and pathological grading $(r=0.310, P<0.001)$, TNM stage $(r=0.155, P=0.003)$, and lymph node metastasis $(r=0.279, P<0.001)$ by using Spearman analysis. In two subgroups of non-small cell lung cancer (NSCLC) and small cell lung cancer (SCLC), the expression of CDK5 was also higher than that of normal lung tissue, respectively $(P=0.001$ and $P=0.004)$. Moreover, in NSCLCs, Spearman analysis revealed that expression of CDK5 was correlated with TNM stages $(r=0.129, P=0.017)$, lymph node metastasis $(r=0.365, P<0.001)$, and pathological grading $(r=0.307, P<0.001)$, respectively. The significant correlation was also found between CDK5 expression and TNM stages $(r=0.415, P=0.049)$ and lymphatic metastasis $(r=0.469, P=0.024)$ in SCLCs.
\end{abstract}

Conclusions: The results of this present study suggest that the CDK5 expression is associated with several clinicopathological factors linked with poorer prognosis.

Keywords: Lung neoplasms, Cyclin-dependent kinase 5, Immunohistochemistry, Tissue array analysis, Neoplasm metastasis

\footnotetext{
*Correspondence: 150871746@qq.com; chen_gang_triones@163.com

${ }^{\dagger}$ Equal contributors

${ }^{2}$ Department of Medical Oncology, First Affiliated Hospital of Guangxi Medical University, No.6 Shuangyong Road, Nanning, Guangxi Zhuang Autonomous Region 530021, People's Republic of China

'Department of Pathology, First Affiliated Hospital of Guangxi Medical University, No.6 Shuangyong Road, Nanning, Guangxi Zhuang Autonomous Region 530021, People's Republic of China
}

C 2016 Wei et al. Open Access This article is distributed under the terms of the Creative Commons Attribution 4.0 International License (http.//creativecommons.org/licenses/by/4.0/, which permits unrestricted use, distribution, and reproduction in any medium, provided you give appropriate credit to the original author(s) and the source, provide a link to the Creative Commons license, and indicate if changes were made. The Creative Commons Public Domain Dedication waiver (http://creativecommons.org/publicdomain/zero/1.0/) applies to the data made available in this article, unless otherwise stated. 


\section{Background}

Lung cancer is the most common type of cancer and the leading cause of cancer-related deaths in the world [1, 2]. In China, the incidence and the mortality of lung cancer increase rapidly, and now, lung cancer is the first dominating cancer [3]. Non-small cell lung cancer (NSCLC) is the most frequent (approximately $85 \%$ ) class of lung cancers [4, 5]. As a result of the insufficiency of efficacious biomarkers for early diagnosis, the majority of lung cancer patients are diagnosed in an advanced stage [6]. Although there are increasing proofs of therapeutic targets like EGFR, HER2, ALK, ROS1, BRAF, MET, VEGF, and FGFR1 and perpetual endeavor in clinic, the prognosis for patients with NSCLC still remains poor, with only a 5-year survival rate of $15 \%$ with the normal therapy [7-9]. Therefore, there is an urgent requirement to discover new stable and independent biomarkers for prognosis and molecular therapy for lung cancer.

Cyclin-dependent kinases (CDKs) are serine/threonine kinases activated by cyclins [10]. CDK5 is a member of CDKs and the investigation of CDK5 in cancer is increasing. In addition to western blot analysis, immunohistochemistry has also been performed to detect expression of CDK5 in cancer tissue [11]. CDK5 has been reported to be upregulated in prostate cancer, breast cancer, medullary thyroid carcinoma, pituitary adenoma, and hepatocellular carcinoma, and CDK5 gene amplification was found in lung cancer [11-16]. However, decreased expression of CDK5 was detected in gastric cancer [17]. The results of the studies showed that CDK5 was greatly related to proliferation, migration, and motility of cancer cells [13-17]. Moreover, downregulation of CDK5 indicated higher overall survival in multiple myeloma [18]. With regard to prognostic implications, decreased expression of CDK5 was associated with advanced clinical stage and poor survival in gastric cancer patients and increased CDK5 expression was correlated to high pathological grading in breast cancer $[11,17]$. So far, several articles have studied the potential role of CDK5 in lung cancer in vitro [19-22]. However, only one paper mentioned the clinical contribution of CDK5 in lung cancer with only 95 NSCLC patients and without small cell lung cancer (SCLC) cases by Liu et al. [23]. In the current study, we set up a larger sample size of 365 lung cancers, 3.8 times bigger than the previous study performed by Liu et al. [23]. Hence, the objective of this study was to explore the expression and clinicopathological significance of CDK5 in lung cancers and investigate its potential role of CDK5 as a biomarker for diagnosis and prognosis prediction for lung cancer patients.

\section{Methods}

\section{Tissue samples}

This study was conducted with 395 samples including 365 lung cancers and 30 normal lung tissues. The fixation was performed shorter than 15 min after surgical removal of the tissue with neutral-buffered formalin (10\%), and fixation time was 24-48 $\mathrm{h}$ according to the tissue size. Two pathologists (Kanglai Wei and Gang Chen) screened all the collected hematoxylin- and eosinstained sections and selected areas of the paraffinembedded tissue specimens that contained representative tumor or non-tumorous cells. Two tissue cores of $0.6 \mathrm{~mm}$ in diameter were taken from each donor block sample and arrayed into a new blank recipient paraffin block (35 $\mathrm{mm} \times 22 \mathrm{~mm} \times 5 \mathrm{~mm}$ ) with a commercially available microarray instrument (Beecher Instruments, USA). Two TMA blocks included 150 cases (300 tissue cores) and the third one comprised of 95 cases (190 tissue cores), respectively. The total lung material was mounted into three blocks. The age range of lung cancer patients was from 19 to 84 years and was from 19 to 73 years of normal lung tissue. The mean age was 57.67 and 54.03 years for cancer and normal controls, respectively. When lung cancer was separated into two subgroups, there were 339 samples of NSCLCs and 26 samples of SCLCs. Furthermore, NSCLCs were composed of 127 samples of adenocarcinomas, which included four subtypes of 83 cases of acinar adenocarcinoma, 19 cases of papillary adenocarcinomas, 18 cases of bronchioloalveolar cell carcinomas, and 7 cases of mucinous carcinomas. NSCLCs also included 175 squamous cell carcinomas, 28 adenosquamous carcinomas, 8 undifferentiated carcinomas, and 1 large cell carcinoma (Table 1). Various clinicopathological factors of patients were collected, including gender, age, pathological grading, TNM stage, lymph node metastasis, tumor size, and distal metastasis. The samples were obtained by random selection of the lung cancer patients by surgery without cancer-related treatment in the First Affiliated Hospital of Guangxi Medical University from January 2010 to December 2012. Approval of this study was achieved from the Ethical Committee of the First Affiliated Hospital of Guangxi Medical University, clinical doctors and patients. Moreover, two pathologists were responsible for the diagnosis, independently.

\section{Immunohistochemistry}

Santa Cruz Biotechnology (Heidelberg, Germany) provided the CDK5 antibody (C-8,sc-173,1:50 dilution) for immunostaining, and a ZSGB Kit (PV-6000, ZSGB, Beijing, China) was used for the secondary antibody at room temperature. CDK5 immunostaining score was determined by both positive rate of stained tumor cells and staining intensity. Concretely, the positive rate of stained 
Table 1 The classification of lung cancer

\begin{tabular}{lll}
\hline \multicolumn{3}{c}{ Cancer subtype of histology } \\
Lung & SCLC & \\
NSCLC & $\begin{array}{l}\text { Squamous cell } \\
\text { carcinomas } \\
\text { Adenosquamous } \\
\text { carcinomas } \\
\text { Large cell carcinoma } \\
\text { Undifferentiated } \\
\text { carcinomas } \\
\text { Adenocarcinoma }\end{array}$ & \\
& & \\
& & Acinar adenocarcinoma \\
& & Papillary adenocarcinomas \\
& Bronchioloalveolar cell \\
& carcinomas \\
& Mucinous carcinomas \\
\hline
\end{tabular}

This table was to classify the subtypes of lung cancer. Lung cancer is consisted of non-small cell lung cancer (NSCLC) and small cell lung cancer (SCLC). NSCLC was composed of squamous cell carcinomas, adenosquamous carcinomas, large cell carcinoma, undifferentiated carcinomas, and adenocarcinoma. Furthermore, adenocarcinoma was composed of acinar adenocarcinoma, papillary adenocarcinomas, bronchioloalveolar cell carcinomas, and mucinous carcinomas

tumor cells and the corresponding score were assigned as follows: 0 (0 \%), 1 (1-25\%), 2 (26-50\%), 3 (51$75 \%)$, and 4 ( (76-100\%). The intensity of CDK5 staining was scored from 0 to 3 , and the detailed standard was as follows: 0 (no staining), 1 (weak staining), 2 (moderate staining), and 3 (strong staining). Samples were scored by the summation of the percentage of CDK5-positive cells and staining intensity. The total score of immunostaining was more than two which was considered as positive expression of CDK5. Immunostaining was assessed and graded independently by two pathologists (Kanglai Wei and Gang Chen).

\section{Statistical analysis}

The statistical analysis was conducted by SPSS 20.0 completely, and $P$ values less than 0.05 were considered statistically significant. The chi-square test was used in the analysis of contrast of two groups, and when it exceeded two groups, Kruskal-Wallis $\mathrm{H}$ test was performed. Further, Spearman analysis was performed to study the relationship between CDK5 expression and clinicopathological characteristics. Moreover, we conducted ROC curve to evaluate the diagnostic significance of CDK5 in lung cancer, and the area under curve (AUC) of CDK5 more than 0.5 was considered significant.

\section{Results}

\section{(CDK5) expression in lung cancer}

CDK5-positive signaling located in the cytoplasm of the tumor cells. Significantly increased expression of CDK5 in lung cancer tissues $(51.5 \%, 188 / 365)$ was found as compared to that in normal lung tissues $(20 \%, 6 / 30, P=0.001$, Table 2, Fig. 1). Furthermore, we conducted ROC curve to evaluate the diagnostic significance of CDK5 in lung cancer. The AUC of CDK5 was 0.685 (95\% CI 0.564 0.751, $P=0.004)$. However, there was no statistical significance between expression of CDK5 in NSCLC and SCLC. Concerning the correlation between $\mathrm{CDK} 5$ expression and clinical features, CDK5 was found to be related to several clinicopathological parameters (Table 3). The positive rate of CDK5 expression was higher $(68.3 \%, 43 / 63)$ in advanced stages (III and IV) than in early stages (I and II) (47.8\%, $143 / 299, P=0.003$, Table 3). Higher expression of CDK5 was found in lung cancer patients with lymphatic metastasis $(76.6 \%, 98 / 128)$ compared to those without lymphatic metastasis $(37.6 \%, 88 / 234, \quad P<0.001$, Table 3). In

Table 2 CDK5 expression in lung cancer compared with normal lung tissue

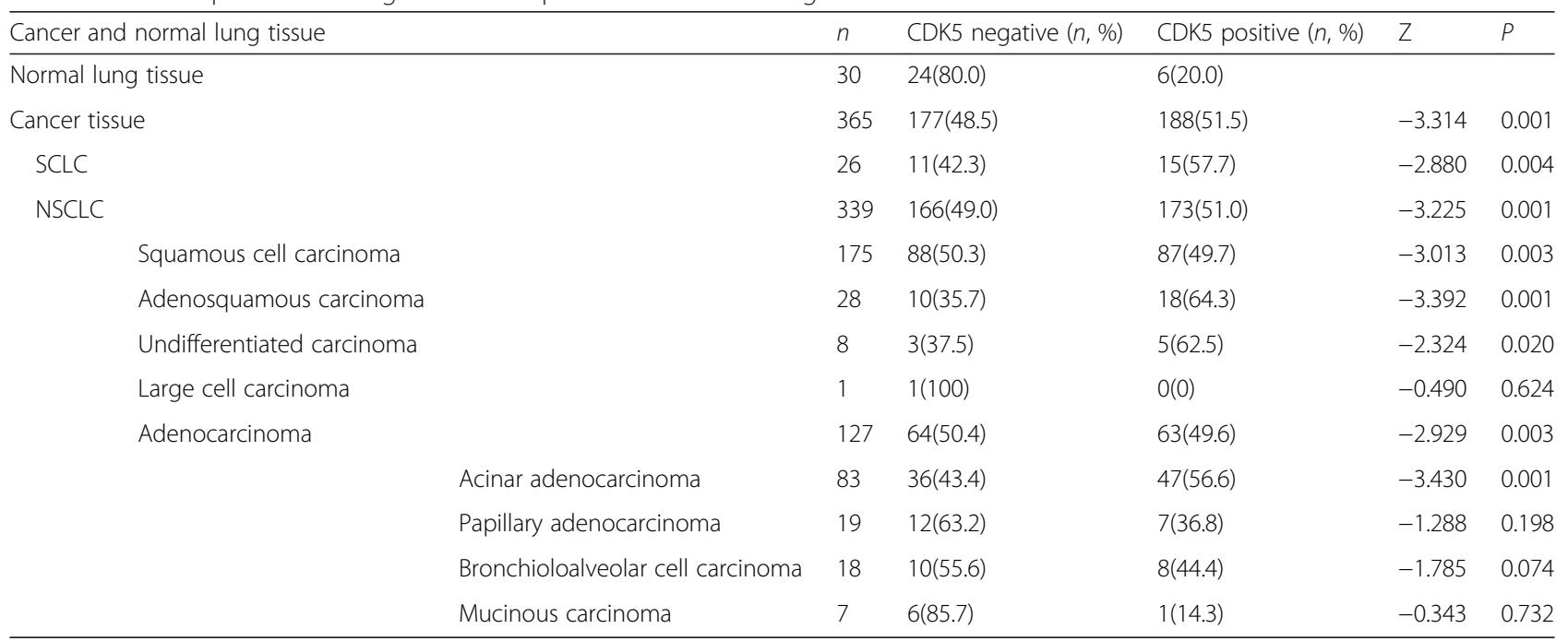




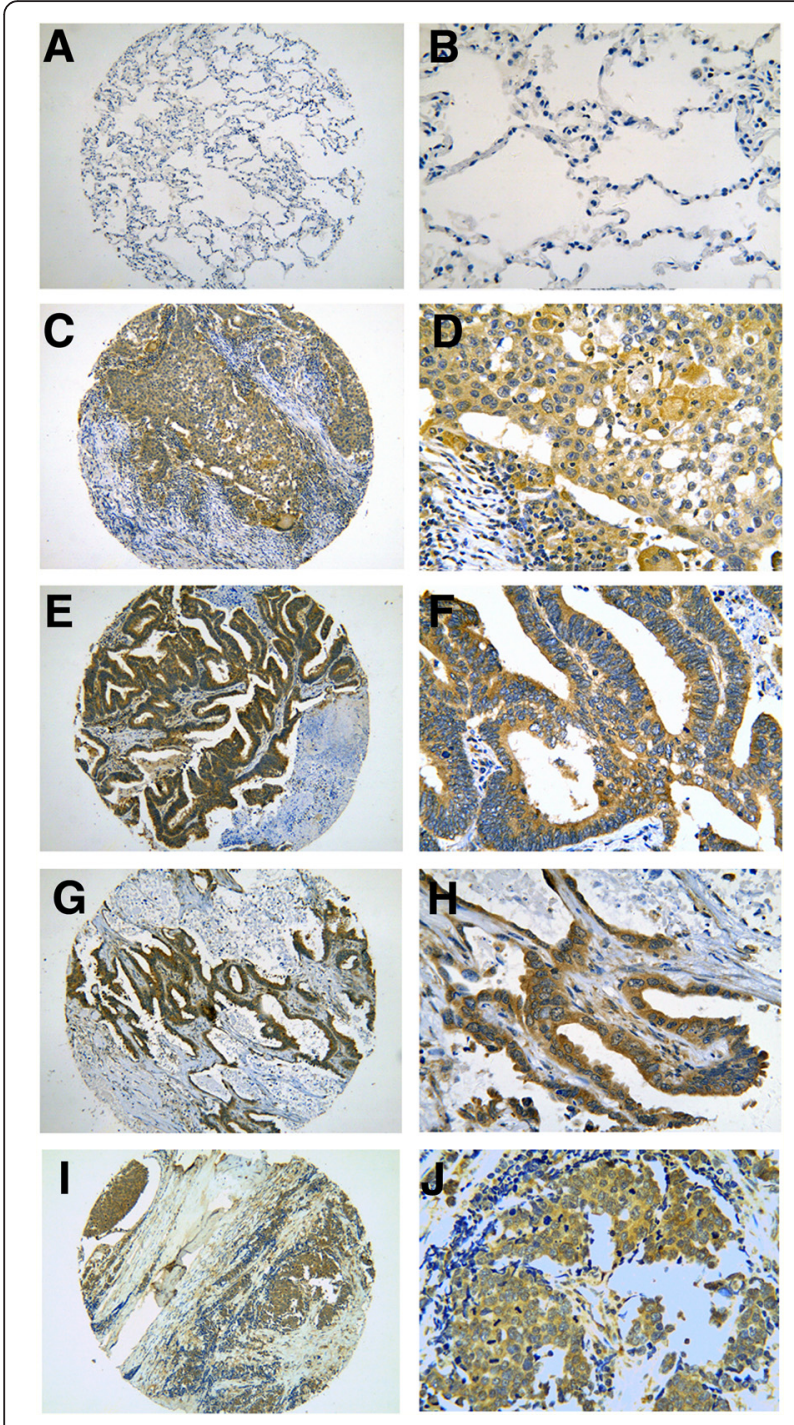

Fig. 1 Immunohistochemical staining of CDK5 in lung tissue. Negative expression of CDK5 was found in normal lung cancer tissue $(\mathbf{a} \times 100$, $\mathbf{b} \times 400)$ and significantly positive expression of CDK5 was detected in the cytoplasm of squamous carcinoma (c $\times 100, \mathbf{d} \times 400)$, papillary adenocarcinoma $(\mathbf{e} \times 100, \mathbf{f} \times 400)$, bronchioloalveolar cell carcinoma $(\mathbf{g} \times 100, \mathbf{h} \times 400)$, small cell lung cancer $(\mathrm{SCLC}, \mathbf{i} \times 100, \mathbf{j} \times 400)$

pathological grading III (66.4 \%, 87/131), CDK5 expression was higher than that in pathological grading I (25.6\%, $10 / 39, P<0.001)$ and there was an increasing trend for CDK5 positive rate as the pathological grading increased $(P<0.001$, Table 3$)$. In addition, Spearman coefficient of correlation was performed to investigate the relationship between the expression of CDK5 and clinicopathological parameters. It was showed that there were close correlations between CDK5 expression and TNM stage $(r=0.155, P=0.003)$, lymph node metastasis $(r=0.279, P<0.001)$, and pathological grading $(r=0.310, P<0.001)$. A marginal correlation between CDK5 expression and distal metastasis has been found $(r=0.102, P=0.053)$. Besides, no significant correlation between CDK5 expression and other clinicopathological factors was discovered, such as gender, age, and tumor diameter.

\section{Cyclin-dependent kinases (CDK5) expression in non-small cell lung cancer (NSCLC)}

When lung cancer patients were separated into two subgroups of NSCLC and SCLC, we discovered that there was higher positive rate in NSCLC compared with normal lung tissues $(P=0.001$, Table 4$)$. Higher CDK5-positive expression was also found in the subgroups of NSCLCs including adenocarcinoma $(P=0.003)$, squamous cell carcinoma $(P=0.003)$, adenosquamous carcinoma $(P=0.001)$, and undifferentiated carcinoma $(P=0.02)$, than that in the normal lung tissue. After adenocarcinoma was further split into four different types, remarkably higher expression of CDK5 was found in acinar adenocarcinoma as compared to normal lung tissues $(P=0.001$, Table 4$)$. When the relationship between CDK 5 expression and other parameters was concerned in the patients with NSCLCs, higher CDK5 expression positive rate appeared in the advanced stages (III and IV) $(66 \%, 35 / 53)$ compared with the early stages (I and II) $(48.3 \%, 138 / 286, P=0.018$, Table 4$)$ and the similar result was found in lymph node metastasis $(76.5 \%, 88 / 115)$ as compared to non-lymph node metastasis (37.9\%, 85/ 224, $P<0.001$, Table 4). In pathological grading III, the positive expression of CDK5 was $66.2 \%(86 / 130)$ in the cases of NSCLC higher than that in pathological grading I (25.6 \%, 10/39)and II (42.4 \%, 39/92, both $P<0.001$, Table 4$)$. Borderline difference of CDK5 expression has been found between distal metastasis $(75 \%, 12 / 16)$ and non-distal metastasis (49.8\%, 161/323, $P=0.05$, Table 4). Moreover, Spearman analysis showed that the positive CDK5 expression in NSCLC was correlated with TNM stages $(r=0.129$, $P=0.017)$, lymph node metastasis $(r=0.365, P<0.001)$, and pathological grading $(r=0.307, P<0.001)$. A marginal correlation between CDK5 expression and distal metastasis was also noticed $(r=0.107, P=0.05)$.

\section{Cyclin-dependent kinases (CDK5) expression in small cell lung cancer (SCLC)}

There were 26 patients of SCLC, and the positive rate of CDK5 expression was $57.7 \%$ (15/26), significant higher compared to normal lung tissues $(20 \%, 6 / 30, P=0.004)$. In the patients with SCLC, higher expression of CDK5 was found in female $(100 \%, 5 / 5)$ and lymph node metastasis (76.9\%, 10/13) compared with that in male (47.6\%, 10/21, $P=0.037)$ and without lymph node metastasis (30 \%, 3/10, $P=0.028$, Table 5), respectively. Spearman coefficient of correlation showed that the positive CDK5 expression in SCLC was correlated with gender $(r=0.418, P=0.034)$, TNM stages $(r=0.415, P=0.049)$, and lymph node metastasis $(r=0.469, P=0.024$, Table 5$)$. 
Table 3 CDK5 expression associated with the various clinicopathological parameters in lung cancer

\begin{tabular}{|c|c|c|c|c|c|}
\hline Lung cancer & $n$ & CDK5 negative $(n, \%)$ & CDK5 positive $(n, \%)$ & $z$ & $P$ \\
\hline Gender & & & & -0.884 & 0.377 \\
\hline Male & 275 & 137(49.8) & $138(50.2)$ & & \\
\hline Female & 90 & $40(44.4)$ & $50(55.6)$ & & \\
\hline Age(years) & & & & -0.418 & 0.676 \\
\hline$<60$ & 196 & $96(49.0)$ & $100(51.0)$ & & \\
\hline$\geq 60$ & 169 & $81(47.9)$ & $88(52.1)$ & & \\
\hline Pathological grading & & & & $25.060^{\mathrm{a}}$ & $<0.001$ \\
\hline । & 39 & $29(74.4)$ & 10(25.6) & & \\
\hline$\|$ & 92 & $53(57.6)$ & $39(42.4)$ & & \\
\hline III & 131 & 44(33.6) & $87(66.4)$ & & \\
\hline TNM & & & & -2.944 & 0.003 \\
\hline$|-| \mid$ & 299 & $156(52.2)$ & $143(47.8)$ & & \\
\hline III-IV & 63 & $20(31.7)$ & $43(68.3)$ & & \\
\hline LNM & & & & -7.080 & $<0.001$ \\
\hline Yes & 128 & $30(23.4)$ & $98(76.6)$ & & \\
\hline No & 234 & $146(62.4)$ & $88(37.6)$ & & \\
\hline Tumor diameter $(\mathrm{cm})$ & & & & -1.653 & 0.098 \\
\hline$\leq 7$ & 314 & $158(50.3)$ & $156(49.7)$ & & \\
\hline$>7$ & 48 & 18(37.5) & $30(62.5)$ & & \\
\hline Distal metastasis & & & & -1.931 & 0.054 \\
\hline Absent & 346 & $172(49.7)$ & $174(50.3)$ & & \\
\hline Present & 16 & $4(25.0)$ & $12(75.0)$ & & \\
\hline
\end{tabular}

${ }^{a}$ Kruskal-Wallis $H$ test was performed between the groups of pathological grading

\section{Discussion}

Cyclin-dependent kinase 5 (CDK5) is vital in neural cell migration and differentiation and is activated by p35 or p39 [24], and CDK5 is considered to be essential in neuronal cells $[25,26]$. Nevertheless, as a unique member of cyclin-dependent kinases, the function of CDK5 beyond the nervous system has been demonstrated. CDK5 also regulates cell proliferation by alterant expression and its downstream signaling pathways, especially in cancer cells. Up to date, there has been a growing number of evidence that CDK5 has an important effect on cancer progression [27]. The expression of CDK5 was aberrant in several cancers, and CDK5 regulated the proliferation of cancer cell in prostate cancer, medullary thyroid carcinoma, and gastric cancer [14, 15, 17]. In breast cancer, CDK5 was essential for the motility of cancer cell [11]. Moreover, CDK5 can be used to predict the prognosis of multiple myeloma [18]. In a word, the role of CDK5 in cancer is attracting increasing attention. To date, the expression of CDK5 was investigated in several cancers [13, 15, 17]. Higher expression of CDK5 was observed in hepatocellular carcinoma, ampullary adenocarcinoma, breast cancer, and medullary thyroid carcinoma, and Zachary et al. confirmed the expression of CDK5 was upregulated in colorectal, head/neck, breast, lung, ovarian, lymphoma, prostatic, sarcoma, myeloma, and bladder cancers via the Oncomine microarray online data mining software [11, 12, 18, 28]. However, downregulated expression of CDK5 was observed in gastric cancer. Thus, CDK5 might be heterogeneously expressed in different cancers.

In the present study, immunohistochemistry on lung tissue microarrays was performed to explore the expression of CDK5 in lung cancer and normal lung tissues. There was prominently higher expression of CDK5 in lung cancer, independent of various pathological subtypes, than in normal lung tissue. In the study of Liu et al., CDK5 was upregulated in cancer tissue as compared to benign pulmonary disease with a sample size of 95 non-small cell lung cancers (NSCLCs) [23]. Our study, with a bigger sample size, approximately four times, confirmed that increased expression of CDK5 could be detected in lung cancer tissue compared with normal lung tissue and further supported that CDK5 was considered as an oncogene in lung cancer. No significant difference of CDK5 expression was found between NSCLC and SCLC in this current study. Although NSCLC and SCLC are commonly regarded as different 
Table 4 The correlation of CDK5 with diverse clinical clinicopathological factors in NSCLC

\begin{tabular}{|c|c|c|c|c|c|}
\hline$\overline{N S C L C}$ & $n$ & CDK5 negative $(n, \%)$ & CDK5 positive $(n, \%)$ & Z & $P$ \\
\hline Gender & & & & -0.406 & 0.685 \\
\hline Male & 254 & $126(49.6)$ & $128(50.4)$ & & \\
\hline Female & 85 & $40(47.1)$ & $45(52.9)$ & & \\
\hline Age(years) & & & & -0.080 & 0.936 \\
\hline$<60$ & 181 & $89(49.2)$ & $92(50.8)$ & & \\
\hline$\geq 60$ & 158 & $77(48.7)$ & $81(51.3)$ & & \\
\hline Pathological grading & & & & $24.58^{\mathrm{a}}$ & $<0.001$ \\
\hline । & 39 & $29(74.4)$ & $10(25.6)$ & & \\
\hline$\|$ & 92 & $53(57.6)$ & $39(42.4)$ & & \\
\hline III & 130 & 44(33.8) & $86(66.2)$ & & \\
\hline TNM & & & & -2.376 & 0.018 \\
\hline$|-| \mid$ & 286 & $148(51.7)$ & 138(48.3) & & \\
\hline III-IV & 53 & 18(34.0) & $35(66.0)$ & & \\
\hline LNM & & & & -6.717 & $<0.001$ \\
\hline Yes & 115 & $27(23.5))$ & $88(76.5)$ & & \\
\hline No & 224 & $139(62.1)$ & 85(37.9) & & \\
\hline Tumor diameter $(\mathrm{cm})$ & & & & -1.145 & 0.252 \\
\hline$\leq 7$ & 295 & $148(50.2)$ & 147(49.8) & & \\
\hline$>7$ & 44 & $18(40.9)$ & $26(59.1)$ & & \\
\hline Distal metastasis & & & & -1.962 & 0.05 \\
\hline Absent & 323 & $162(50.2)$ & $161(49.8)$ & & \\
\hline Present & 16 & $4(25.0)$ & 12(75.0) & & \\
\hline Histology & & & & $3.646^{\mathrm{a}}$ & 0.456 \\
\hline Adenocarcinoma & 127 & 64(50.4) & 63(49.6) & & \\
\hline Squamous cell carcinoma & 175 & $88(50.3)$ & $87(49.7)$ & & \\
\hline Adenosquamous carcinoma & 28 & $10(35.7)$ & 18(64.3) & & \\
\hline Undifferentiated carcinoma & 8 & $3(37.5)$ & $5(62.5)$ & & \\
\hline Large cell carcinoma & 1 & $1(100)$ & $0(0)$ & & \\
\hline Adenocarcinoma classification & & & & $6.508^{\mathrm{a}}$ & 0.089 \\
\hline Acinar adenocarcinoma & 83 & $36(43.4)$ & $47(56.6)$ & & \\
\hline Papillary adenocarcinoma & 19 & $12(63.2)$ & $7(36.8)$ & & \\
\hline Broncholoalveolar cell carcinoma & 18 & 10(55.6) & $8(44.4)$ & & \\
\hline Mucinous carcinoma & 7 & $6(85.7)$ & $1(14.3)$ & & \\
\hline
\end{tabular}

Pathological grading I vs. II $Z=-1.805, P=0.071$, I vs. III $Z=-4.466, P<0.001$, II vs. III $Z=-3.508, P<0.001$. Acinar adenocarcinoma vs. mucinous $Z=-2.144$, $P=0.032$. There were no differences of expression of CDK5 in other subgroups

${ }^{a}$ Kruskal-Wallis $\mathrm{H}$ test was performed when the data were divided into more than two groups

diseases owing to their distinct biology and genomic abnormalities, the role and function of CDK5 may be consistent, as CDK5 level was both upregulated in NSCLC and SCLC tissues than the non-cancerous lung. However, the exact role of CDK5 in SCLC needs further investigation, since only a limited sample size $(n=26)$ was included in the current study. Taken together, CDK5 might be a potential biomarker of lung cancer despite its histology types.
The regulative mechanism of CDK5 in several cancers was investigated. CDK5 regulates DNA damage response via phosphorylating Ataxia telangiectasia mutated (ATM) kinase and thereby affecting its downstream signal pathways which was crucial to progression of hepatocellular carcinoma [12]. In ampullary adenocarcinoma, over expression of nestin/CDK5 was involved in several oncogenic pathways (the activation of NOTCH, TGF- $\beta 1$, or PDGFR pathways) that facilitated invasiveness of 
Table 5 The correlation of CDK5 expression with various clinical pathological factors in SCLC

\begin{tabular}{|c|c|c|c|c|c|}
\hline $\mathrm{SCLC}$ & $n$ & CDK5 negative $(n, \%)$ & CDK5 positive $(n, \%)$ & $Z$ & $P$ \\
\hline Gender & & & & -2.089 & 0.037 \\
\hline Male & 21 & $11(52.4)$ & $10(47.6)$ & & \\
\hline Female & 5 & $0(0)$ & $5(100)$ & & \\
\hline Age(years) & & & & -0.515 & 0.606 \\
\hline$<60$ & 15 & $7(46.7)$ & $8(53.3)$ & & \\
\hline$\geq 60$ & 11 & $4(36.4)$ & $7(63.6)$ & & \\
\hline TNM & & & & -1.948 & 0.051 \\
\hline$|-| \mid$ & 13 & $8(61.5)$ & $5(38.5)$ & & \\
\hline III-IV & 10 & $2(20.0)$ & $8(80.0)$ & & \\
\hline LNM & & & & -2.201 & 0.028 \\
\hline Yes & 13 & $3(23.1)$ & 10(76.9) & & \\
\hline No & 10 & $7(70.0)$ & $3(30.0)$ & & \\
\hline Tumor diameter (cm) & & & & -1.888 & 0.059 \\
\hline$\leq 7$ & 19 & 10(52.6) & $9(47.4)$ & & \\
\hline$>7$ & & $0(0)$ & $4(100)$ & & \\
\hline
\end{tabular}

cancer [28]. In breast cancer, CDK5 takes part in epithelial-mesenchymal transition induced by TGF$\beta 1$ which is vital for tumor metastasis [11]. In medullary thyroid carcinoma, CDK5 is essential to tumorigenesis and progression by retinoblastoma protein $(\mathrm{Rb})$ and inhibition of $\mathrm{Rb}$ reduced proliferation of medullary thyroid carcinoma [15]. The mechanisms of tumorigenesis and progression in lung cancer might be similar to the mechanisms aforementioned in other cancers in consideration of a consistent trend of CDK5 expression. However, this hypothesis needs to be verified with in vitro and in vivo studies.

Though expression of CDK5 was detected in lung cancer tissue and regulative mechanism of CDK5 was investigated in other cancers, the mechanism and exact role of CDK5 in the carcinogenesis and development of lung cancer remain unclear. A study of Korean population shows that CDK5 promoter polymorphisms contribute to the genetic susceptibility to lung cancer [20]. As one of the downstream components of the EGFR-familysignaling pathway, the gene of CDK5 was amplified in lung cancer and it might be the common mechanism of oncogene activation in carcinogenesis [16]. Tripathi et al. demonstrated that the CDK5 phosphorylates four serines located N-terminal to the Rho-GTPase activating protein(Rho-GAP) domain in DLC1(deleted in lung cancer 1), a tumor suppressor protein, and thereby activates DLC1 [22]. Through the reconstruction of an integrated genome-scale co-expression network, Bidkhori et al. exhibited that CDK5 played a vital role in cell cycle progression in lung adenocarcinoma [19]. The studies above of CDK5 in lung cancer suggested CDK5 may play an oncogenic role in lung cancer.
In addition to the expression of CDK5 in cancer tissues, the relationship between CDK5 and pathological parameters has been paid more and more attention to. There were a few researches of the relationship between CDK5 and clinical factors in the patients with cancers. In breast cancer, upregulated expression of CDK5 was related to higher grading (grading III) [11]. In multiple myeloma, downregulated expression of cdk5 predicted favorable overall survival after bortezomib treatment [18]. Liu et al. demonstrated that higher expression of CDK5 was correlated with low/ undifferentiated, high pathological stage, lymph node metastasis, shorter median survival, and lower 5-year overall survival in the patients with NSCLC [23]. Similarly, with a larger sample size, the consistent trend in this current study was confirmed that higher positive rate of CDK5 expression was greatly correlated with unfavorable clinicopathological parameters, including advanced TNM stage, lymphatic metastasis, and high pathological grading, which commonly indicate poorer prognosis. Thus, CDK5 might be used for the prediction to prognosis of lung cancer.

Further, Demelash et al. [21] demonstrated that CDK5 played a vital role in the regulation of lung cancer cell migration and invasion through Wound closure and Boyden chamber assay and certified that achaete-scute homologue-1 (ASH1), a basic transcription factor which was expressed in lung cancer cells with neuroendocrine features [29], could stimulate migration of lung cancer cells through CDK5/ p35 pathway. The mechanism may support that CDK5 was closed related to lymphatic metastasis in lung cancer. 


\section{Conclusions}

In summary, in this current study, the expression of CDK5 was investigated by lung tissue microarrays and immunohistochemistry. We demonstrated that CDK5 was highly expressed in lung cancer, including non-small cell lung cancer and small cell lung cancer, compared to normal lung tissue. Higher positive rate of CDK5 was associated with several clinicopathological parameters, which are representative of the progression and deterioration of lung cancer. These results suggest that the CDK5 expression associated with several unfavorable clinicopathological factors linked with poorer prognosis. Nevertheless, further plans are needed to explore the potential function of CDK5 in vitro and in vivo in the carcinogenesis of and progression in lung cancer.

\section{Competing interests}

The authors declare that they have no competing interests.

\section{Authors' contributions}

KLW and ZYL designed the present study. YWD, LHY, and GC were responsible for immunohistochemistry and analysis of data. ZHY and XC conducted the experiment and wrote the paper. $\mathrm{NH}, \mathrm{CXB}$, and TQG contributed to statistical analysis. All authors read and approved the final manuscript.

\section{Acknowledgements}

The study was supported by the Fund of Guangxi Zhuang Autonomous Region University Student Innovative Plan (no. WLXSZX1555), China, the Fund of National Natural Science Foundation of China (NSFC 81360327), and the fund of Guangxi Provincial Health Bureau Scientific Research Project (Z2013201, Z2014055). The funders had no role in the study design, data collection and analysis, decision to publish, or preparation of the manuscript.

Received: 30 July 2015 Accepted: 26 January 2016

Published online: 09 February 2016

\section{References}

1. Barrow TM, Michels KB. Epigenetic epidemiology of cancer. Biochem Biophys Res Commun. 2014;455(1-2):70-83. doi:10.1016/j.bbrc.2014.08.002

2. Siegel RL, Miller KD, Jemal A. Cancer statistics, 2015. CA Cancer J Clin. 2015:65(1):5-29. doi:10.3322/caac.21254.

3. Chen W, Zheng $\mathrm{R}$, Zhang S, Zhao P, Zeng H, Zou X, et al. Annual report on status of cancer in China, 2010. Chin J Cancer Res. 2014:26(1):48-58. doi:10.3978/j.issn.1000-9604.2014.01.08.

4. Brothers JF, Hijazi K, Mascaux C, El-Zein RA, Spitz MR, Spira A. Bridging the clinical gaps: genetic, epigenetic and transcriptomic biomarkers for the early detection of lung cancer in the post-national lung screening trial era. BMC Med. 2013;11:168. doi:10.1186/1741-7015-11-168.

5. Jafri SH, Shi R, Mills G. Advance lung cancer inflammation index (ALI) at diagnosis is a prognostic marker in patients with metastatic non-small cell lung cancer (NSCLC): a retrospective review. BMC Cancer. 2013;13:158. doi:10.1186/1471-2407-13-158.

6. Zhang C, Huang C, Wang J, Wang X, Li K. Maintenance or consolidation therapy for non-small-cell lung cancer: a meta-analysis involving 5841 subjects. Clin Lung Cancer. 2015. doi:10.1016/j.cllc.2015.01.002

7. DeSantis CE, Lin CC, Mariotto AB, Siegel RL, Stein KD, Kramer JL, et al. Cancer treatment and survivorship statistics, 2014. CA Cancer J Clin. 2014:64(4):252-71. doi:10.3322/caac.21235.

8. Barr Kumarakulasinghe N, Zanwijk NV, Soo RA. Molecular targeted therapy in the treatment of advanced stage non-small cell lung cancer (NSCLC). Respirology. 2015. doi:10.1111/resp.12490

9. Parums DV. Current status of targeted therapy in non-small cell lung cancer. Drugs Today. 2014;50(7):503-25. doi:10.1358/dot.2014.50.7.2185913.

10. Gerard C, Tyson JJ, Coudreuse D, Novak B. Cell cycle control by a minimal cdk network. PLoS Comput Biol. 2015;11(2):e1004056. doi:10.1371/journal. pcbi.1004056
11. Liang Q, Li L, Zhang J, Lei Y, Wang L, Liu DX, et al. CDK5 is essential for TGFbeta1-induced epithelial-mesenchymal transition and breast cancer progression. Scientific Reports. 2013;3:2932. doi:10.1038/srep02932.

12. Ehrlich SM, Liebl J, Ardelt MA, Lehr T, De Toni EN, Mayr D et al. Targeting cyclin dependent kinase 5 in hepatocellular carcinoma-a novel therapeutic approach. J Hepatol. 2015. doi:10.1016/j.jhep.2015.01.031

13. Xie W, Wang H, He Y, Li D, Gong L, Zhang Y. CDK5 and its activator P35 in normal pituitary and in pituitary adenomas: relationship to VEGF expression. Int J Biol Sci. 2014;10(2):192-9. doi:10.7150/ijbs.7770.

14. Lindqvist J, Imanishi SY, Torvaldson E, Malinen M, Remes M, Orn F et al. Cyclin-dependent kinase 5 acts as a critical determinant of AKT-dependent proliferation and regulates differential gene expression by the androgen receptor in prostate cancer cells. Molecular biology of the cell. 2015. doi:10.1091/mbc.E14-12-1634

15. Pozo K, Castro-Rivera E, Tan C, Plattner F, Schwach G, Siegl V, et al. The role of Cdk5 in neuroendocrine thyroid cancer. Cancer Cell. 2013;24(4):499-511. doi:10.1016/j.ccr.2013.08.027.

16. Lockwood WW, Chari R, Coe BP, Girard L, Macaulay C, Lam S, et al. DNA amplification is a ubiquitous mechanism of oncogene activation in lung and other cancers. Oncogene. 2008:27(33):4615-24. doi:10.1038/onc.2008.98.

17. Cao L, Zhou J, Zhang J, Wu S, Yang X, Zhao X et al. Cyclin dependent kinase 5 decreases in gastric cancer and its nuclear accumulation suppresses gastric tumorigenesis. Clin Cancer Res. 2015. doi:10.1158/10780432.CCR-14-1950

18. Levacque Z, Rosales JL, Lee KY. Level of cdk5 expression predicts the survival of relapsed multiple myeloma patients. Cell Cycle. 2012;11(21):40935. doi:10.4161/cc.21886.

19. Bidkhori G, Narimani Z, Hosseini Ashtiani S, Moeini A, Nowzari-Dalini A, Masoudi-Nejad A. Reconstruction of an integrated genome-scale co-expression network reveals key modules involved in lung adenocarcinoma. PLoS One. 2013;8(7):e67552. doi:10.1371/journal.pone. 0067552 .

20. Choi HS, Lee Y, Park KH, Sung JS, Lee JE, Shin ES, et al. Single-nucleotide polymorphisms in the promoter of the CDK5 gene and lung cancer risk in a Korean population. J Hum Genet. 2009;54(5):298-303. doi:10.1038/jhg.2009. 29.

21. Demelash A, Rudrabhatla P, Pant HC, Wang X, Amin ND, McWhite CD, et al. Achaete-scute homologue-1 (ASH1) stimulates migration of lung cancer cells through Cdk5/p35 pathway. Mol Biol Cell. 2012;23(15):2856-66. doi:10.1091/mbc.E10-12-1010.

22. Tripathi BK, Qian X, Mertins P, Wang D, Papageorge AG, Carr SA, et al. CDK5 is a major regulator of the tumor suppressor DLC1. J Cell Biol. 2014;207(5): 627-42. doi:10.1083/jcb.201405105.

23. Liu JL, Wang XY, Huang BX, Zhu F, Zhang RG, Wu G. Expression of CDK5/ p35 in resected patients with non-small cell lung cancer: relation to prognosis. Med Oncol. 2011;28(3):673-8. doi:10.1007/s12032-010-9510-7.

24. Shah K, Lahiri DK. Cdk5 activity in the brain-multiple paths of regulation. J Cell Sci. 2014:127(Pt 11):2391-400, doi:10.1242/jcs.147553.

25. Nishimura Y, Shikanai M, Hoshino M, Ohshima T, Nabeshima Y, Mizutani K, et al. Cdk5 and its substrates, Dcx and p27kip1, regulate cytoplasmic dilation formation and nuclear elongation in migrating neurons. Development. 2014;141(18):3540-50. doi:10.1242/dev.111294.

26. Xu S, Li X, Gong Z, Wang W, Li Y, Nair BC, et al. Proteomic analysis of the human cyclin-dependent kinase family reveals a novel CDK5 complex involved in cell growth and migration. Mol Cell Proteomics. 2014;13(11): 2986-3000. doi:10.1074/mcp.M113.036699.

27. Kimura T, Ishiguro K, Hisanaga S. Physiological and pathological phosphorylation of tau by Cdk5. Front Mol Neurosci. 2014;7:65. doi:10.3389/fnmol.2014.00065.

28. Shan YS, Chen YL, Lai MD, Hsu HP. Nestin predicts a favorable prognosis in early ampullary adenocarcinoma and functions as a promoter of metastasis in advanced cancer. Oncol Rep. 2015;33(1):40-8. doi:10.3892/or.2014.3588.

29. Miki M, Ball DW, Linnoila RI. Insights into the achaete-scute homolog-1 gene (hASH1) in normal and neoplastic human lung. Lung Cancer. 2012; 75(1):58-65. doi:10.1016/j.lungcan.2011.05.019. 